\title{
A Note on Standard Goal Programming with Fuzzy Hierarchies: A Sequential Approach
}

\author{
Maged George Iskander \\ Faculty of Business Administration, Economics and Political Science, The British University in Egypt, \\ El-Sherouk City, Egypt \\ Email: maged.iskander@bue.edu.eg
}

Received 19 November 2015; accepted 23 January 2016; published 27 January 2016

Copyright (C) 2016 by author and Scientific Research Publishing Inc.

This work is licensed under the Creative Commons Attribution International License (CC BY).

http://creativecommons.org/licenses/by/4.0/

c) (i) Open Access

\begin{abstract}
In the paper [Standard goal programming with fuzzy hierarchies: a sequential approach, Soft Computing, First online: 22 March 2015], it was assumed that the normalized deviations should lie between zero and one. In some cases, this assumption may not be valid. Therefore, additional constraints must be incorporated into the model to ensure that the normalized deviations should not exceed one. This modification is illustrated by the given numerical example.
\end{abstract}

\section{Keywords}

Fuzzy Goal Programming, Imprecise Hierarchy, Normalized Deviations

\section{Introduction}

The problem of fuzzy goal programming when the importance relation between the fuzzy goals is vague has been investigated by Aköz and Petrovic [1] and followed by $\mathrm{Li}$ and $\mathrm{Hu}$ [2] and Cheng [3]. A suggested sequential approach in fuzzy goal programming, when the importance hierarchy of the goals is imprecise, has been presented by Arenas-Parra et al. [4]. In their article, the model of goal programming with fuzzy hierarchy $(\mathrm{GPFH})$ is given as

$$
\text { Maximize } \lambda \sum_{i=1}^{k}\left(1-\frac{n_{i}}{m_{i}-\underline{f_{i}}}\right)+(1-\lambda) \sum_{\substack{(i, j)=1 \\ i \neq j}}^{k} \sum_{r=1}^{3} b_{\tilde{R}_{r}(i, j)} \mu_{\tilde{R}_{r}(i, j)}
$$

subject to: 


$$
\begin{aligned}
& f_{i}(x)+n_{i}-p_{i}=m_{i}, \quad i=1, \cdots, k, \\
& 1-\left(\frac{n_{i}}{m_{i}-\underline{f_{i}}}-\frac{n_{j}}{m_{j}-\underline{f_{j}}}\right) \geq \mu_{\tilde{R}_{1}(i, j)}, \quad \text { if } b_{\tilde{R}_{1}(i, j)}=1, \\
& \frac{1-\left(\frac{n_{i}}{m_{i}-\underline{f_{i}}}-\frac{n_{j}}{m_{j}-\underline{f_{j}}}\right)}{2} \geq \mu_{\tilde{R}_{2}(i, j)}, \quad \text { if } b_{\tilde{R}_{2}(i, j)}=1, \\
& \frac{n_{j}}{m_{j}-\underline{f_{j}}}-\frac{n_{i}}{m_{i}-\underline{f_{i}}} \geq \mu_{\tilde{R}_{3}(i, j)}, \quad \text { if } b_{\tilde{R}_{3}(i, j)}=1, \\
& 0 \leq \mu_{\tilde{R}_{r}(i, j)} \leq 1, r=1,2,3, \\
& n_{i}, p_{i} \geq 0, n_{i} \times p_{i}=0, \quad i=1, \cdots, k, \\
& x \in X
\end{aligned}
$$

where $0 \leq \lambda \leq 1$, and $f_{i}(x)$ is the $i^{t h}$ linear function of the $x$ vector of decision variables, $i=1, \cdots, k$. Also, $n_{i}$ and $p_{i}$ are the negative and positive deviations, respectively, while $m_{i}$ is the aspiration level and $f_{i}$ is the anti-ideal value for the $i^{\text {th }}$ fuzzy goal constraint. Moreover, $b_{\tilde{R}(i, j)}(r=1,2,3)$ is a binary variable associated with the membership function of the $r^{\text {th }}$ importance relation (slightly, moderately, significantly) of the $i^{\text {th }}$ goal more than the $j^{\text {th }}$ goal; while $\mu_{\tilde{R}_{r}(i, j)}$ is the membership function of the $r^{\text {th }}$ imprecise relation between the $i^{\text {th }}$ and the $j^{\text {th }}$ fuzzy goals. Finally, $X$ is a set of system constraints which define the feasible set of the problem.

This model is implemented for each class of Phase I. Hence, it is assumed that the normalized deviation for the $i^{\text {th }}$ fuzzy goal constraint must lie between zero and one i.e.,

$$
0 \leq n_{i} /\left(m_{i}-\underline{f_{i}}\right) \leq 1 .
$$

This assumption may be violated, especially when the anti-ideal value is close to the aspiration level. In this case, $n_{i} /\left(m_{i}-\underline{f_{i}}\right)$ may exceed one, due to a small denominator value, which means that the value of the achieved goal is worse than the anti-ideal value of that goal. Accordingly, for each class, the following constraints should be incorporated in the GPFH model:

$$
n_{i} \leq m_{i}-\underline{f_{i}},
$$

if the negative deviation is required to be minimized for the $i^{\text {th }}$ fuzzy goal constraint, i.e., if $f_{i}(x) \geq m_{i}$; or

$$
p_{i} \leq \underline{f_{i}}-m_{i},
$$

if the positive deviation is required to be minimized for the $i^{\text {th }}$ fuzzy goal constraint, i.e., if $f_{i}(x) \leq m_{i}$.

Notably, constraints (3) and (4) correspond to the non-negativity of the membership functions of the fuzzy goal constraints given by Aköz and Petrovic [1].

Proposition: The constraints of the normalized deviations might limit the feasible set of the problem. This may worsen the value of the achievement function of each class and, therefore, affect the results of the suggested sequential approach.

In the next section, this note is verified by the given illustrative example.

\section{Illustrative Example}

The GPFH model (Phase I) is solved using the following example that is given by Arenas-Parra et al. [4]:

Goal 1: $4 x_{1}+2 x_{2}+8 x_{3}+x_{4} \leq 35$

Goal 2: $4 x_{1}+7 x_{2}+6 x_{3}+2 x_{4} \geq 100$

Goal 3: $x_{1}-6 x_{2}+5 x_{3}+10 x_{4} \geq 120$ 
Goal 4: $5 x_{1}+3 x_{2}+2 x_{4} \geq 70$

Goal 5: $4 x_{1}+4 x_{2}+4 x_{3} \geq 40$

subject to:

$$
\left.\begin{array}{l}
7 x_{1}+5 x_{2}+3 x_{3}+2 x_{4} \leq 98, \\
7 x_{1}+x_{2}+2 x_{3}+6 x_{4} \leq 117, \\
x_{1}+x_{2}+2 x_{3}+6 x_{4} \leq 130, \\
9 x_{1}+x_{2}+6 x_{4} \leq 105, \\
x_{i} \geq 0, i=1, \cdots, 4,
\end{array}\right\} X
$$

where Class $I$ contains goals (1, 2, and 4). Accordingly, the assumed anti-ideal values for these goals are $f_{1}=261.33, f_{2}=0, f_{4}=0$. Also, the GPFH model for Class $I$ assumes that Goal 1 is moderately more important than Goal 2; and Goal 2 is moderately more important than Goal 4. Finally, the parameter $\lambda_{I}$ is set equal to 0.8 .

Thus, the model for Class I is as follows:

$$
\text { Maximize } A F_{I}=\lambda_{I}\left(1-\frac{p_{1}}{226.33}+1-\frac{n_{2}}{100}+1-\frac{n_{4}}{70}\right)+\left(1-\lambda_{I}\right)\left[\mu_{\tilde{R}_{2}(1,2)}+\mu_{\tilde{R}_{2}(2,4)}\right]
$$

subject to:

$$
\begin{aligned}
& 4 x_{1}+2 x_{2}+8 x_{3}+x_{4}+n_{1}-p_{1}=35, \\
& 4 x_{1}+7 x_{2}+6 x_{3}+2 x_{4}+n_{2}-p_{2}=100, \\
& 5 x_{1}+3 x_{2}+2 x_{4}+n_{4}-p_{4}=70, \\
& \frac{1-\left(\frac{p_{1}}{226.33}-\frac{n_{2}}{100}\right)}{2} \geq \mu_{\tilde{R}_{2}(1,2)}, \\
& \frac{1-\left(\frac{n_{2}}{100}-\frac{n_{4}}{70}\right)}{2} \geq \mu_{\tilde{R}_{2}(2,4)}, \\
& 0 \leq \mu_{\tilde{R}_{2}(1,2)} \leq 1, \quad 0 \leq \mu_{\tilde{R}_{2}(2,4)} \leq 1, \\
& n_{k}, p_{k} \geq 0, \quad n_{k} \times p_{k}=0, \quad k=1,2,4, \\
& x \in X .
\end{aligned}
$$

The given note is verified by just resolving the GPFH model for Class I in Phase I. Assume that the anti-ideal values of the first and the fourth fuzzy goal constraints $\underline{f}_{1}$ and $f_{4}$ are 40 and 63 instead of 261.33 and 0, respectively. In this case, the normalized $p_{1}$ is $p_{1} / 5$, while the normalized $n_{4}$ becomes $n_{4} / 7$.

Then, the solution obtained is: $\mu_{\tilde{R}_{2}(1,2)}=0.463, \mu_{\tilde{R}_{2}(2,4)}=1, p_{1}=0.375, n_{2}=0, n_{4}=9, G_{1}=35.375, G_{2}=100$, $G_{4}=61, A F_{I}^{*}=1.604$. Hence, $n_{4} / 7=1.286$, which is greater than 1 .

Accordingly, by incorporating the following three constraints:

$$
\begin{aligned}
& p_{1} \leq 5, \\
& n_{2} \leq 100, \\
& n_{4} \leq 7,
\end{aligned}
$$

and by solving the model, the solution becomes: $\mu_{\tilde{R}_{2}(1,2)}=0.325, \mu_{\tilde{R}_{2}(2,4)}=1, p_{1}=1.750, n_{2}=0, n_{4}=7, G_{1}=$ 36.750, $G_{2}=105, G_{4}=63, A F_{I}^{*}=1.585$.

It is realized that incorporating the constraints of the normalized deviations leads to a worse value of $A F_{I}^{*}$, which verifies the proposition. 


\section{Conclusion}

The constraints of the normalized deviations must be included in the GPFH model in all classes of Phase I as well as in Phase II to ensure that the achieved value of each goal should never become worse than the anti-ideal value of that goal.

\section{References}

[1] Aköz, O. and Petrovic, D. (2007) A Fuzzy Goal Programming Method with Imprecise Goal Hierarchy. European Journal of Operational Research, 181, 1427-1433. http://dx.doi.org/10.1016/j.ejor.2005.11.049

[2] Li, S. and Hu, C. (2009) Satisfying Optimization Method Based on Goal Programming for Fuzzy Multiple Objective Optimization Problem. European Journal of Operational Research, 197, 675-684. http://dx.doi.org/10.1016/j.ejor.2008.07.007

[3] Cheng, H.-W. (2013) A Satisficing Method for Fuzzy Goal Programming Problems with Different Importance and Priorities. Quality and Quantity, 47, 485-498. http://dx.doi.org/10.1007/s11135-011-9531-0

[4] Arenas-Parra, M., Bilbao-Terol, A. and Jiménez, M. (2015) Standard Goal Programming with Fuzzy Hierarchies: A Sequential Approach. Soft Computing. http://dx.doi.org/10.1007/s00500-015-1644-2 